\title{
Patients of advanced maternal age should only transfer a single euploid blastocyst
}

\author{
Catherine Gordon ${ }^{1}$, John B Whitney ${ }^{2}$, Ilene Hatch ${ }^{3}$, Nancy L Nugent ${ }^{2}$, Shane Zozula ${ }^{2}$, Robert E Anderson ${ }^{4}$ and Mitchel C Schiewe $^{2 *}$ \\ ${ }^{1}$ University of California-Irvine, Department of OB - GYN, Orange, CA 92868, USA \\ ${ }^{2}$ Ovation Fertility/SCIRS, ART Lab, Newport Beach, CA 92663, USA \\ ${ }^{3}$ Fertility Center of Southern California, Irvine, CA 92612, USA \\ ${ }^{4}$ Southern California Center for Reproductive Medicine, Newport Beach, CA 92663, USA
}

\begin{abstract}
Background: The IVF industry has been trying to reduce high order multiple pregnancies by promoting single embryo transfer for nearly two decades. Although improvements in embryo culture practices concurrently occurred, poor prognosis patients and those of advanced maternal age ( $\geq 38$ years old) proved to be challenging cases when determining the number of embryos to transfer and yet still optimize pregnancy success. It was not until preimplantation genetic screening (PGS) of blastocysts was coupled with conservative embryo transfer decisions that worldwide progress occurred. The objective of this study was to determine the efficacy of single embryo transfer (SET) compared to dual embryo transfer (DET) in older patients (age $\geq 38$ ) performing vitrified-warmed, euploid ET cycles.

Methods and findings: Retrospective cohort analysis was performed on 140 vitrified-warmed euploid blastocyst transfers of patients $\geq 38$ years old performing either a SET ( $n=122)$ or DET ( $n=18)$. All full to hatched blastocysts were initially biopsied on Days 5, 6 or 7, and the trophectoderm samples were analyzed using NGS or aCGH. All transfers represented the patients first transfer attempt following PGS between January 2013 to June 2015 . Implantation and live birth results per ET treatment were evaluated and compared using Chi-squared analysis $(\mathrm{p}<0.05)$. The average patient age was 39.7 years old, achieving a clinical pregnancy rate of $83 \%$ $(116 / 140)$ and a live birth rate of $80 \%(112 / 140)$. SET achieved a live birth rate of $79.5 \%(97 / 122)$ similar to DET (15/18, 83.3\%). Although pregnancy outcome comparisons were not different between age groups or treatments, a trend $(\mathrm{p}<0.10)$ toward higher implantation for SET was observed. Most significantly, the twinning rate was appreciably higher $(\mathrm{p}<0.001)$ with DET at $73 \%(11 / 15)$ compared to $1 \%$ for SET $(1 / 97)$.
\end{abstract}

Conclusions: Independent of age, when using euploid blastocysts, we believe that SET should be adopted as the standard of care for clinics utilizing PGS. This is especially true for the first ET attempt by patients of advanced maternal age to optimize implantation rates and reduce the potential wastage of precious euploid embryos.

\section{Introduction}

Today's human assisted reproductive technology (ART) industry strives to increase live birth rates, while decreasing the occurrence of multiple gestations. In turn, the current clinical focus is on how to best offer couples the option of transferring only one embryo to decrease the chance of a multiple pregnancy. High order multiple pregnancies, including twins, are associated with increased pregnancy complications and numerous perinatal risks $[1,2]$, especially in women of advanced maternal age ( $\geq 38$ years old). Numerous studies have advocated for elective single embryo transfer (eSET) given a $90-95 \%$ reduction in multiple gestations [3-5]. While some programs experienced a 20 to $40 \%$ reduction in live birth rates with eSET [3], other studies using improved embryo culturing practices showed no effect to a conservative ET approach [6,7]. As such, much of the literature argues in favor of SET only in optimal population groups [8], such as those patients age $<35$ years old and with $>2$ good quality blastocysts for ET [9]. Fujimoto and coworkers [7] recently reported that eSET actually improved live birth rates in patients age $\leq 37$ years old, but not in older patients. It has also been reported that vitrification-all ET cycles can improve pregnancy outcomes by transferring embryos into a more progesterone synchronized, receptive uterine environment [10].

Considering the current move toward SET, importance has been placed on embryo selection. Subjective morphology grading has been the commonly accepted standard to judge embryo quality [11], but our ability to select embryos with high success of implantation has been limited by solely judging morphology [12]. Other recent developments using time-lapsed imaging (TLI) have shown promise for improving the selection process [13]. Yet the latter methods, morphologic grading and TLI, are not highly predictive of genetic normalcy once a blastocyst is produced. The application of preimplantation genetic screening (PGS), incorporating genomic array technologies, to confirm embryo euploidy status prior to transfer has proven to be a highly effective approach for embryo selection [14,15]. The clinical application of blastocyst biopsy/PGS practices has led to decreases in spontaneous abortion and multiple gestations, and overall improvements in live birth rates [5,14-16]. In fact, it has now been shown that the quality grading of blastocysts (>fair to good quality; 3BB or better) does not correlate to euploidy predictability [17].

Correspondence to: Mitchel C. Schiewe, PhD; 361 Hospital Road, Suite 433; Newport Beach, USA, Tel: +01-310-4898775; Fax: +01-310-8207394; E-mail: mschiewe@ovationfertility.com

Key words: blastocyst, preimplantation genetic screening, single embryo transfer, euploidy

Received: March 01, 2018; Accepted: March 13, 2018; Published: March 16, 2018 
Prior to 2017, ET guidelines of the American Society for Reproductive Medicine recommended the transfer of 2 or 3 blastocysts in patients of advanced maternal age. The diminished ovarian reserve and increased aneuploidy rates of this patient population typically limits the number of viable embryos produced per cycle. The problem lies in knowing which, if any, embryo(s) are genetically normal. While multiple embryos transferred increases live birth success [4,6], it does come at the risk of establishing multiple gestations in a higher pregnancy risk population. The aim of this study was to determine the appropriate number of euploid embryos to transfer in an older IVF patient population undergoing IVF/PGS treatment.

\section{Materials and methods}

\section{Embryo culture, grading, biopsy, and PGS}

Using MCO-5M mini Sanyo/Panasonic tri-gas incubators (5\% 02/5.3-6.0 \% $\left.\mathrm{CO}_{2}\right)$ under humidified air conditions $\left(37^{\circ} \mathrm{C}\right)$, we group cultured up to five embryos per $25 \mu \mathrm{L}$ droplet of Global $^{\text {Ts }}$ medium (LG; Life Global, Guilford, CT) supplemented with $7.5 \%$ synthetic protein supplement under Ovoil ${ }^{\text {Ts }}$ (Vitrolife, Englewood, CO) until blastocyst biopsy $[17,18]$. All oocytes retrieved were evaluated for maturity and had ICSI performed $2-6$ h post-egg retrieval. Embryos were initially evaluated on Day 3; laser zona dissection was performed using a 1480-nm diode laser (Zilos-tk ${ }^{\mathrm{rw}}$; Hamilton Thorne, Beverly, MA), and embryo incubation continued until Days 5, 6 and possibly Day 7 for evaluation and biopsy determination $[5,18]$. The zona opening created on Day 3 allowed trophectoderm (TE) to prematurely rupture through a $10-12-\mu \mathrm{m}$ furrow in the zona. Blastocysts were graded at biopsy using a modified Gardner scale [11]. The modification was necessary to account for premature hatching: grade $3=<10 \%$ TE extrusion (full blastocyst), grade $4=10 \%-50 \%$ TE extrusion (expanded blastocyst), and grade $5 \geq 50 \% \mathrm{TE}$ extrusion (hatching blastocyst)[5,18]. Inner cell mass (ICM) and TE were independently graded from top quality " $A$ " to fair quality "B" and poor quality " $C$ " with the first letter in the grade assigned to the ICM and the second to TE. A grade of $3 \mathrm{BB}$ or better was required to initiate biopsy. All residual developing embryos were allowed to continue in vitro culture to Day 7 for possible late biopsy consideration. The diode laser was again used on Day 5/6/7 for biopsying, combining laser pulse ablation and mechanical aspiration to separate 3-10 TE cells [5]. All TE samples were aseptically tubed, frozen and shipped to Genesis Genetics (Plymouth, MI) or Ovation Fertility Genetics (Henderson, NV) for array CGH or NGS analysis.

\section{Vitrification and embryo transfer}

Fair to excellent quality blastocysts ( $\geq 3 \mathrm{BB}$ grade) were vitrified on Days 5,6 or 7 using microSecure-VTF in glycerol based, non-DMSO vitrification (VTF) solutions (Innovative Cryo Enterprises, Linden, NJ; 19). Aseptic microSecure VTF was performed using a 3-step dilution ( $5 \mathrm{~min} / 5 \mathrm{~min} / 1 \mathrm{~min}$ ); individual blastocysts were loaded into $300 \mu \mathrm{m}$ ID flexipettes (Cook Medical, Spencer, IL; $3 \mu$ volume); flexipettes were then dried and inserted tip first into prelabeled $0.3 \mathrm{ml} \mathrm{CBS}{ }^{\mathrm{m}}$ embryo straws; the straws were weld sealed and plunged directly into $\mathrm{LN}_{2}[19,20]$. Rapid warming was achieved by direct placement of the vitrified flexipettes into a warm $\left(37^{\circ} \mathrm{C}\right) 0.5 \mathrm{M}$ sucrose bath [21]. Within 10 seconds, each blastocyst was pipette directly from the flexipette into an open $200 \mu$ droplet of $1.0 \mathrm{M}$ sucrose solution and then transferred into $100 \mu \mathrm{l}$ droplets under oil for $3 \mathrm{~min}$ intervals. Embryos were serially diluted in declining sucrose solutions (T1-T4), before isotonic equilibration in Hepes-LG medium. Warmed blastocysts were then cultured in LG medium + protein for 1-3 h prior to vitrified ET (VFET).
All VFET's involved hormone replacement cycles using oral estradiol, estradiol patches, or intramuscular (IM) estradiol valerate followed by IM progesterone in oil. Progesterone in oil was started when endometrial thickness was $>8 \mathrm{~mm}$ after documentation of serum progesterone level of $<1.0 \mathrm{ng} / \mathrm{ml}$. VFET was performed after 5.5 days of IM progesterone administration. Transvaginal ultrasound guidance ET procedures were performed. Pregnancies were initially tested 10 days post-ET and implantation subsequently assessed by transvaginal ultrasound beginning 4 weeks later. Live births were confirmed by written or oral communication with patients.

\section{Study design and statistical analysis}

Using an observational retrospective cohort analysis, we strived to determine the efficacy of SET compared to DET in advanced maternal age patients (age $\geq 38$ ) when preimplantation genetic screening is applied. We evaluated 158 frozen euploid embryo transfer cycles between January 2013 and June 2015 from the Southern California Institute for Reproductive Sciences (SCIRS) medical records. Inclusion criteria for the study was simply the production of at least 1 euploid blastocyst graded BB and above $(n=140)$, based on a recent study validating implantation potential of euploid blastocysts [5]. Only VFET cycles were performed and all donor egg/embryo cycles were excluded. Initial comparisons for implantation, clinical pregnancies, live births, and spontaneous abortions were calculated per first transfer attempt. Chi-squared analyses were performed to contrast differences in pregnancy outcomes (e.g., clinical pregnancy, implantation, live birth rates, twin production) between the SET and DET groups. Clinical pregnancy determination required cardiac activity confirmation, whereas implantation rates included any sac detected by ultrasound exam.

\section{Results}

The average age of 140 qualified study patients was 39.7 years old, with no difference detected between groups. The overall clinical pregnancy rate was $83 \%(116 / 140)$ and a live birth rate of $80 \%(112 / 140)$ selectively transferring euploid blastocysts. When comparing SET to DET, live birth rates ( $79.5 \%$ and $83.3 \%$, respectively) were not statistically different. However, SET implantation rates (82.9\%) trended toward being higher $(\mathrm{p}<0.10)$ compared to DET $(75 \%)$. Our inability to detect significant differences overall and between age groups (Table 1) is attributed to the low sample size in the DET groups and high level of pregnancy success achieved overall. Not surprisingly, the twinning rate was appreciably higher $(\mathrm{p}<0.001)$ with DET at $73 \%(11 / 15)$ in contrast to a single identical twin birth following SET in this study $(1 / 97,1 \%)$.

\section{Discussion}

While initial studies recommended SET only in ideal patient populations, age $<35$ and with $>2$ good quality embryos [6,9], we believe SET should be used as a first line attempt in patients of every age with known fair-to-good quality euploid blastocysts. When contrasting SET to DET of euploid blastocysts, both protocols yielded similar live birth outcomes for women of advanced maternal age. More importantly, the risk of twinning was virtually eliminated by transferring a single euploid embryo. Conversely, transferring two euploid blastocysts resulted in an exceptionally high twin rate. Although most infertile patients and couples would welcome the blessing of two babies, our goal has always been a single, healthy term birth. Twin pregnancies have a six-fold increased risk of premature delivery and three-fold increase in perinatal mortality compared to singleton pregnancies, as well as elevated risks of maternal complications including hypertensive disorders and hemorrhaging $[1,2]$. 
Table 1. Euploid SET versus DET pregnancy outcomes for patients of advanced maternal age.

\begin{tabular}{|c|c|c|c|c|c|c|}
\hline \multirow{2}{*}{$\begin{array}{c}\text { Age } \\
\text { (years old) }\end{array}$} & \multicolumn{3}{|c|}{ SET } & \multicolumn{3}{|c|}{ DET } \\
\hline & Implantation & Clinical Preg & Live Birth & Implantation & Clinical Preg & Live Birth \\
\hline $38-40$ & $\begin{array}{l}79.1 \% \\
72 / 91\end{array}$ & $\begin{array}{l}79.1 \% \\
72 / 91\end{array}$ & $\begin{array}{c}75.8 \% \\
69 / 91\end{array}$ & $\begin{array}{c}68.8 \% \\
11 / 16\end{array}$ & $\begin{array}{c}87.5 \% \\
7 / 8\end{array}$ & $\begin{array}{c}87.5 \% \\
7 / 8\end{array}$ \\
\hline $41-42$ & $\begin{array}{l}93.1 \% \\
27 / 29\end{array}$ & $\begin{array}{l}93.1 \% \\
27 / 29\end{array}$ & $\begin{array}{l}89.7 \% \\
26 / 29\end{array}$ & $\begin{array}{l}88.9 \% \\
16 / 18\end{array}$ & $\begin{array}{c}88.9 \% \\
8 / 9\end{array}$ & $\begin{array}{c}88.9 \% \\
8 / 9\end{array}$ \\
\hline $43+$ & $\begin{array}{l}100 \% \\
2 / 2\end{array}$ & $\begin{array}{c}100 \% \\
2 / 2\end{array}$ & $\begin{array}{c}100 \% \\
2 / 2\end{array}$ & $\begin{array}{l}0 \% \\
0 / 2\end{array}$ & $\begin{array}{l}0 \% \\
0 / 1\end{array}$ & $\begin{array}{l}0 \% \\
0 / 1\end{array}$ \\
\hline Total & $\begin{array}{c}82.7 \% \\
101 / 122\end{array}$ & $\begin{array}{c}82.7 \% \\
101 / 122\end{array}$ & $\begin{array}{l}79.5 \% \\
97 / 122\end{array}$ & $\begin{array}{l}75.0 \% \\
27 / 36\end{array}$ & $\begin{array}{c}83.3 \% \\
15 / 18\end{array}$ & $\begin{array}{l}83.3 \% \\
15 / 18\end{array}$ \\
\hline
\end{tabular}

Abbreviations: SET - single embryo transfer; DET - dual embryo transfer.

Fundamentally, when embryos are screened genetically normal, their implantation potential outperforms the need to transfer more than one embryo. This data validates our decision to adopt SET as standard clinical practice for all first ET attempts using euploid blastocysts. PGS-ET cycles have been previously shown to increase implantation and live birth rates (50.9\% and $45.5 \%$, respectively) in women $40-43$ years old [22], granted at a lower level than observed in our laboratory. Interestingly, a trend was noted favoring increased implantation rates in the SET group compared to DET. This may hint toward a possible adverse competition between embryos during implantation, however we have previously documented in vitro that blastocyst amalgamation can occur [18]. The latter event suggests that trophectodermal cells are highly compatible with other blastocysts and are indeed programmed toward cellular invasion by Day 6, even with one another on occasion $(0.015 \%)$. Overall, our retrospective analysis was limited by a small DET sample size, but with a larger patient population we believe that these implantation rates differences would convey significance.

It is our clinical practice to promote blastocyst biopsy/PGS cycles for patients of advanced maternal age, knowing that the rate of embryo aneuploidy is progressively higher in this population [23]. Our experience is similar to other recent reports with euploidy rates ranging from $25.2-35.3 \%$ at $38-40$ years old, $15.9-20.5 \%$ at $41-42$ years old and $17-23.1 \%$ at $>43$ years old (Ovation Fertility, unpublished data 2017 ). Consequently, $24 \%, 42 \%$ and $81 \%$ of these patients, respectfully, will fail to produce euploid blastocysts for a subsequent VFET cycle. Yet, our approach provides optimism that we are offering patients an informative, direct, more emotionally balanced path to pregnancy success. Undoubtedly, PGS does add cost to the cycle, but one must not discount the enormous cost of emotional distress and trauma endured by women experiencing repeated failures and fetal loss transferring morphologically good quality, aneuploid embryos. Furthermore, fetal losses can potentially complicate the patients' future fertility, as well as delay their ability to become pregnant again. Time is a precious commodity in the older age population evaluated in this study. We feel the ability to diagnose potential cycle failures and drastically reduce the rate of pregnancy losses in women $\geq 38$ years old (i.e., SAB; 25 $75 \%$ in untested ET cycles), strongly justifies blastocyst biopsy/PGS intervention. Factoring in the limited pregnancy loss and increased healthy singleton live births associated with euploid SET, we are now able to offer a more enjoyable, safer and positive IVF experience for the majority of our patients.

\section{Acknowledgments}

The authors would like to thank the Embryology team at Ovation Fertility, formally SCIRS, for working so well together in providing their technical expertise for embryo culturing, biopsy, and vitrification which has facilitated the reliable, elite results generated by our laboratory.

\section{Conflicts of interest}

Dr. Robert Anderson and John Whitney serve as ART medical and technical advisors, respectively, to Illumina. All other authors have no commercial affiliations to declare.

\section{References}

1. Rao A, Sairam S, Shehata H (2004) Obstetric complications of twin pregnancies. Best Pract Res Clin Obstet Gynaecol 18: 557-576. [Crossref]

2. Chughtai AA, Wang AY, Hilder L, Li Z, Lui K, et al. (2018) Gestational age-specific perinatal mortality rates for assisted reproductive technology (ART) and other births. Hum Reprod 33: 320-327. [Crossref]

3. Gelbaya TA, Tsoumpou I, Nardo LG (2010) The likelihood of live birth and multiple birth after single versus double embryo transfer at the cleavage stage: a systematic review and meta-analysis. Fertil Steril 94: 936-945. [Crossref]

4. McLernon DJ, Harrild K, Bergh C, Davies MJ, de Neubourg D, et al. (2010) Clinical effectiveness of elective single versus double embryo transfer: meta-analysis of individual patient data from randomised trials. BMJ 341: c6945. [Crossref]

5. Whitney JB, Schiewe MC, Anderson RE. (2016) Single center validation of routine blastocyst biopsy implementation. J Asst Reprod Genet 33: 1507-1513. [Crossref]

6. Gardner DK, Surrey E, Minjarez D, Leitz A, Stevens J, et al. (2004) Single blastocyst transfer: a prospective randomized trial. Fertil Steril 81: 551-555. [Crossref]

7. Fujimoto A, Morishima K, Harada M, Hirata T, Osuga Y, et al. (2015) Elective singleembryo transfer improves cumulative pregnancy outcome in young patients but not in women of advanced reproductive age. J Asst Reprod Genet 32: 1773-1779. [Crossref]

8. Yazbeck C (2011) Elective single embryo transfer for all patients? Mid East Fert Soc 16: $182-188$.

9. Min JK, Hughes E, Young D, Joint SOGC-CFAS clinical practice guidelines committee Reproductive endocrinology and infertility committee (2010) Elective single embryo transfer following in vitro fertilization. J Obstet Gynaecol Can 32: 363-377. [Crossref]

10. Zhu D, Zhang J, Cao S, Zhang J, Heng BC, et al. (2011) Vitrified-warmed blastocyst transfer cycles yield higher pregnancy and implantation rats compared with fresh blastocyst transfer cycles-time for a new embryo transfer strategy? Fertil Steril 95: 1691-1695. [Crossref]

11. Gardner DK (1998) Changes in requirements and utilization of nutrients during mammalian preimplantation embryo development and their significance in embryo culture. Theriogenology 49: 83-102. [Crossref]

12. Ebner T, Vanderzwalmen P, Shebi O, Urdl W, Moder M, et al. (2009) Morphology of vitrified /warmed day-5 embryos predicts rates of implantation, pregnancy and live birth. Reprod BioMed Online 19:72-78. [Crossref]

13. Kramer YG, Kfinas JD, Melzer K, Noyes N, McCaffrey C, et al. (2014) Assessing morphokinetic parameters via time lapse microscopy (TLM) to predict euploidy: are aneuploidy risk classification models universal? J Asst Reprod Genet 31: 1231-1242. [Crossref]

14. Scott Jr RT, Upham KM, Forman EJ, Hong KH, Scott KL, et al. (2013) Blastocyst biopsy with comprehensive chromosome screening and fresh embryo transfer significantly increases in vitro fertilization implantation and delivery rates: a randomized controlled trial. Fertil Steril 100: 697-703. [Crossref]

15. Grifo JA, Hodes-Wertz B, Lee HL, Amperloquio E, Clarke-Williams M, et al. (2013) Single thawed euploid embryo transfer improves IVF pregnancy, miscarriage, and multiple gestation outcomes and has similar implantation rates as egg donation. J Asst Reprod Genet 30: 259-264. [Crossref] 
16. Forman EJ, Hong KH, Ferry KM, Tao X, Taylor D, et al. (2013) In vitro fertilization with single euploid blastocyst transfer: a randomized controlled trial. Fertil Steril 100: 100-107. [Crossref]

17. Whitney JB, Anderson RE, Nugent NL, Schiewe MC (2015) Euploidy predictability of human blastocyst inner cell mass and trophectoderm grading. Annals Clin Lab Res 3: 4-7.

18. Schiewe MC, Whitney JB, Anderson RE (2015) Potential risk of monochorionic dizygotic twin blastocyst formation associated with early laser zona dissection of group cultured embryos. Fertil Steril 103: 417-421. [Crossref]

19. Schiewe MC, Zozula S, Anderson RE, Fahy GM (2015) Validation of microSecure vitrification (mS-VTF) for the effective cryopreservation of human embryos and oocytes. Cryobiol 71: 264-272. [Crossref]
20. Schiewe MC (2010) Microsecure vitrification ( $\mu \mathrm{S}-\mathrm{VTF}$ ) procedure: optimum simplicity, security, cost-saving and effectiveness combining FDA-approved products. J Clin Embryol 13: 33-41.

21. Schiewe MC, Zozula S, Nugent N, Waggoner K, Borba J, et al. (2017) Modified microSecure vitrification: A safe, simple and highly effective cryopreservation procedure for human blastocysts. $J$ Vis Exp 121: e54871, doi:10.3791/54871 (video). [Crossref]

22. Lee HL, McCulloh DH, Hodes-Wertz B, Adler A, McCaffrey C, et al. (2015) In vitro fertilization with preimplantation genetic screening improves implantation and live birth in women age 40 through 43. J Asst Reprod Genet 32: 435-444. [Crossref]

23. Gat I, AlKudmani B, Wong K, Zohni K, Weizman NF, et al. (2017) Significant correlation between anti-müllerian hormone and embryo euploidy in a subpopulation of infertile patients. Reprod Biomed Online 35: 602-608. [Crossref]

Copyright: $\odot 2018$ Gordon C. This is an open-access article distributed under the terms of the Creative Commons Attribution License, which permits unrestricted use, distribution, and reproduction in any medium, provided the original author and source are credited. 